\title{
Miniature Liquid Crystal Lens Optimizations
}

\section{Dieter Cuypers $^{1}$, Rik Verplancke ${ }^{1}$, and Herbert De Smet ${ }^{1}$}

\author{
${ }^{1}$ Centre for Microsystems Technology, imec and Ghent University, Technologiepark 126, B-9052 Ghent, \\ Belgium \\ Keywords: liquid crystal; tunable lens; Fresnel
}

\section{ABSTRACT}

Small, switchable liquid crystal based polymer Fresnel lenses are discussed, considering design optimizations for performance.

\section{INTRODUCTION}

\subsection{Liquid crystal lenses}

Switchable liquid crystal based lenses were introduced already quite some time ago $[1,2]$ but regained interest again in more recent times. Applications where such lenses are very useful include in principle all space restricted environments such as smartphones, medical uses such as endoscopes or intra-ocular lenses and wearables such as contact lenses.

Obviously, all these applications actually feature miniature lenses, with diameters around $10 \mathrm{~mm}$ or less. Larger liquid crystal lenses are of course also possible, but the nature and status of the technology lends itself best to the production of small diameter lenses and this naturally leads to the aforementioned niche applications.

Furthermore, the small diameter lenses and the typically desired focal lengths for the envisaged systems

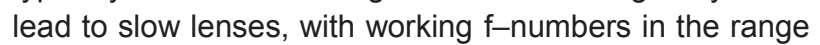
of 10 or more. Exploiting this fact can result in easy to fabricate, relatively simple lens designs that still perform up to par and achieve high optical quality [3].

\subsection{Segmented Fresnel lenses}

The base lens design that best exploits the high $\mathrm{f}$ number is the segmented Fresnel lens. In essence, these are basically sectioned conventional lenses relying entirely on refraction. The thin, polymer based, switchable liquid crystal based variant of such a lens consists of a polymer foil on which the Fresnel segments are manufactured, with nematic, non-twisted liquid crystal on top of them and a second foil to seal the cell.

In figure 1, the according working principle is illustrated. Assuming planar alignment, in the unpowered state a mismatch between the ordinary refractive index of the LC and the index of the lens shape material will give the intended focusing power. With an electric field applied, the liquid crystal molecules will gradually reorient themselves to the vertical position. As such, the mismatch of the refractive indies will reduce until, with correctly selected materials the extraordinary index of the liquid crystal equals the index of the lens material and refraction no longer occurs.
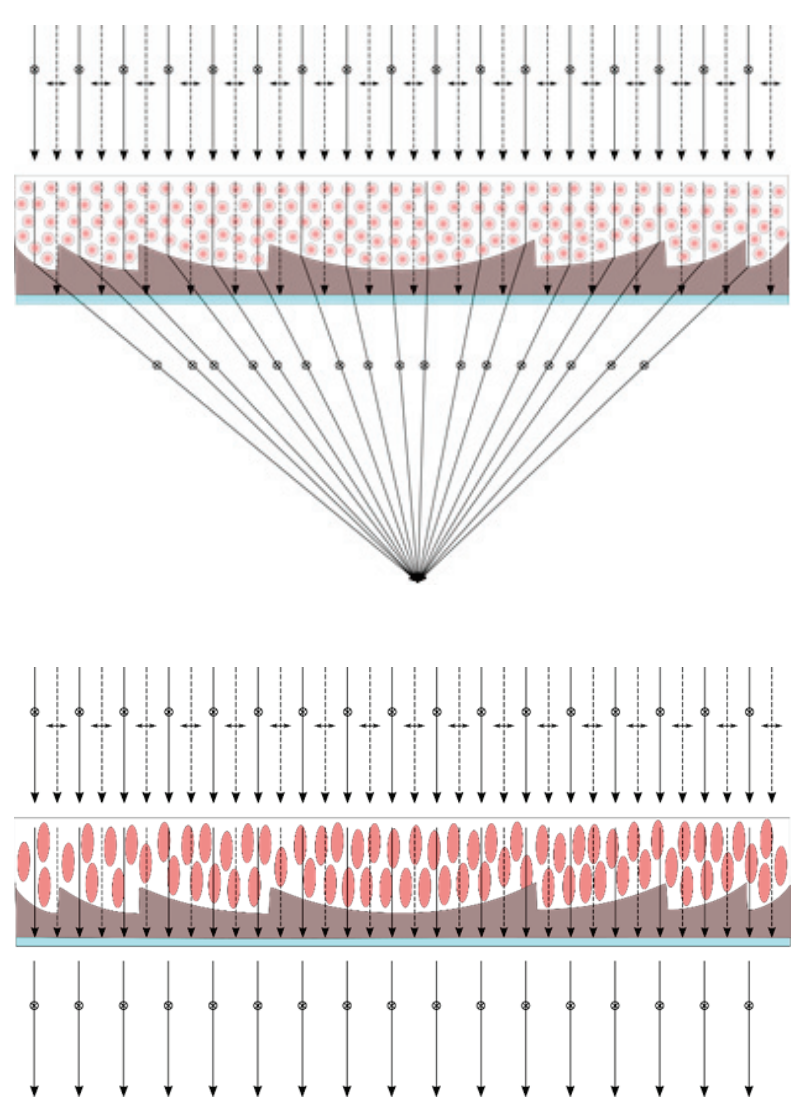

Fig. 1 Basic liquid crystal Fresnel lens working principle.

In the unpowered state (top) the LC molecules are aligned planar. The mismatch between the extraordinary refractive index of the LC and the index of the lens material will then cause refraction to the focal point for one polarization, the other is blocked by the polarizer (light blue) beneath the lens. When a voltage is applied (bottom) the LC molecules reorient to the vertical position. If the ordinary refractive index of the LC and the index of the lens material have been chosen to match, no refraction occurs and the light simply passes through. 


\section{OPTIMIZATIONS}

\subsection{General}

One of the key benefits of a Fresnel lens is its low profile height. This not only guarantees a very compact lens, for liquid crystal lenses it also ensures a thin liquid crystal layer, a crucial parameter to maintain the switching speed as high as possible.

Conventionally, Fresnel lenses can be constructed with either a constant pitch or a constant height for the segments. The latter option is, in view of the above statement and also for ease of manufacturing the preferred option. The manufacturing itself is done using a soft-lithography technique where the structures are imprinted in a UV-curable material [4].

The small diameter and high f-number creates the favorable environment where the segment height can be kept as low as a few micrometers while the lateral dimensions of the segment are in the range of a few hundred micrometers, resulting in only ten to twenty segments and very faint segment slopes [3].

\subsection{Liquid crystal alignment}

The three factors listed above (limited segment height, low segment number and faint slopes) also play a crucial role in the ease of alignment of the liquid crystal.

With Fresnel lenses, the alignment layer for the liquid crystal must be applied over the topography constituted by the segments. Uniform coating of polymer alignment layers is not straightforward under such circumstances and minimal features are then most welcome. Moreover, the linear alignment brought about with the rubbing technique is not really compatible with the circular symmetry of the Fresnel segments. Although some artefacts remain inevitable, low profile heights at least alleviate the problem.

Alternatively, inorganic alignment layers applied with the oblique evaporation technique offer a way of completely removing all artefacts. Application of the layer is in principle contactless, eliminating any problems with coverage uniformity. Once more, the limited slope and height of the structures ensure that shadow effects and incidence angle variations associated with the oblique coating are suppressed. Figure 2 illustrates the resulting alignment quality with this technique.

\subsection{Continuously variable focus distance}

Tunable lenses are usually optimized for one specific optical power and are then switched between two nominal powers (one of which is then provided by the surrounding encapsulation and which can of course be zero). This has the advantage of simplicity in both optical design and electrical driving. The two states then correspond to the unpowered state and a fully switched liquid crystal, which means the driving voltage does not need to be an exact value and only must be chosen high enough. However, intermediate effective refractive indices for the liquid crystal layer can be obtained with proper electrical driving which should in turn yield intermediate focusing distances. In principle, since optimization of the single lens surface can only yield optimum results for one configuration, optimal performance at intermediate focal distances is not a priori guaranteed.

Nonetheless, the performance at intermediate levels does not seem to deteriorate to any appreciable level. Figures 3 to 6 show a number of key characteristics of the lens with the liquid crystal switched to different effective refractive indices. Accounting for the parameters that are directly linked to the changed focal distance, it can be seen that performance remains very much constant.

\section{CONCLUSIONS}

Tunable liquid crystal lenses based on the Fresnel design have been discussed, showing their adequacy and performance level when working at high f-numbers.

\section{REFERENCES}

[1] D.W. Berreman, "Variable Focus Liquid Crystal Lens System". U.S. Patent No. 4,190,330, 26 February (1980).

[2] S. Sato, "Liquid-crystal lens-cells with variable focal length". Jpn. J. Appl. Phys., 18, 1679-1694, (1979)

[3] D. Cuypers, "Design of Active Liquid Crystal Based Contact Lenses", SID 2019 Digest, pp. 985 (2019)

[4] X. Shang, J.-Y. Tan, J. De Smet, P. Joshi, E. Islamaj, D. Cuypers, M. Vervaeke, J. Van Erps, H. Thienpont, H. De Smet, "Replicating micro-optical structures using soft embossing technique", Proc. SPIE 9231, 30th European Mask and Lithography Conference, 92310H (2014)

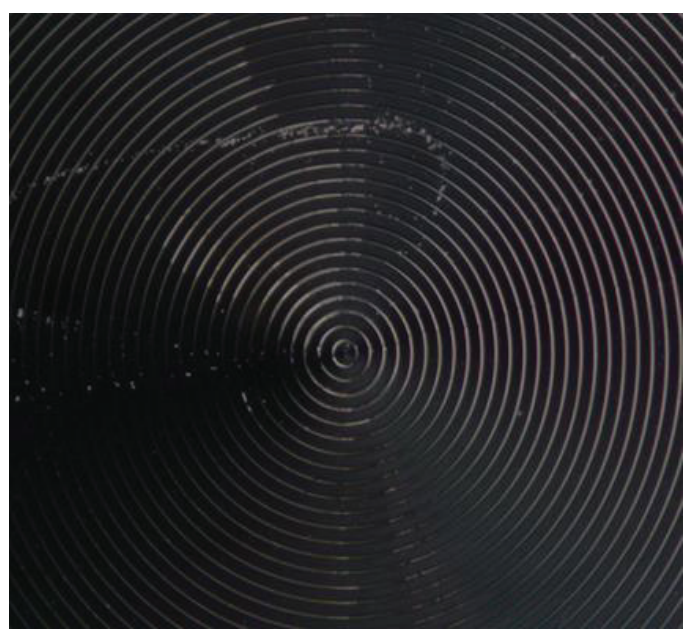

Figure 2: Liquid crystal alignment quality with obliquely evaporated inorganic alignment layers on a circularly symmetric structure.

Micrograph through crossed polarizers with vertically aligned liquid crystal. 

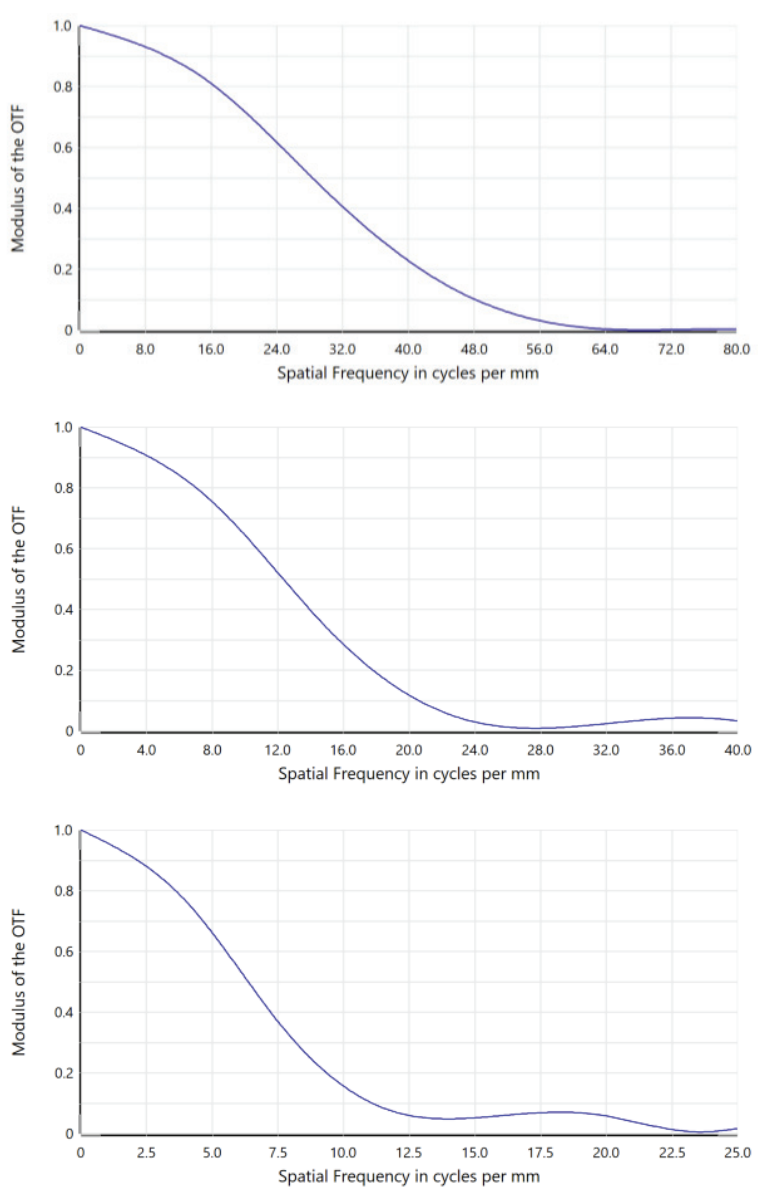

Figure 3: Calculated Modulation Transfer Function for a nominal $125 \mathrm{~mm}$ focal distance lens for varying effective refractive indices.

Evaluation at $100 \%, 50 \%$ and $25 \%$ (top to bottom) of the refractive index difference. Note the differing horizontal scale. Focal distance increases with lower refractive index difference, accounting for the lower MTF numbers.
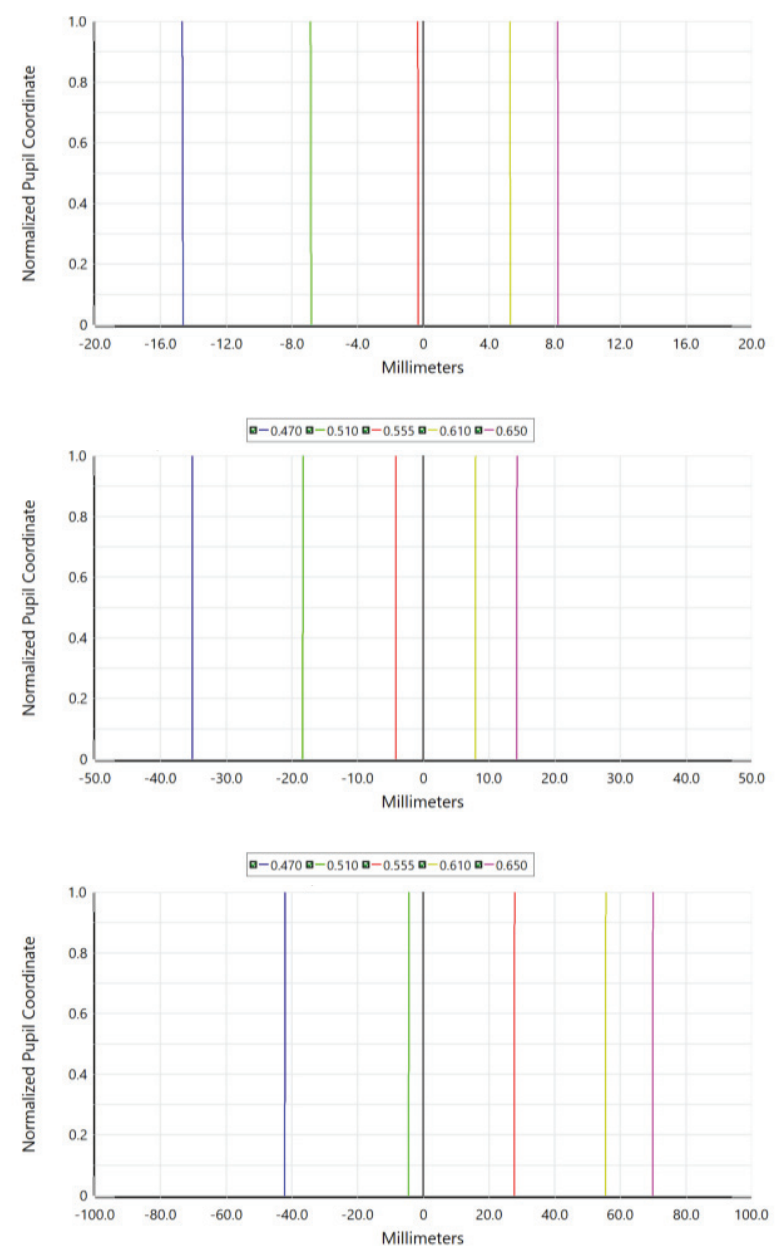

$\mathbf{a}-0.470 \mathrm{a}-0.510 \mathrm{a}-0.555 \mathrm{a}-0.610 \mathrm{a}-0.650$

Figure 4: Longitudinal aberration for a nominal 125 $\mathrm{mm}$ focal distance lens for varying effective refractive indices.

Evaluation at $100 \%, 50 \%$ and $25 \%$ (top to bottom) of the refractive index difference. Wavelengths in micrometer. 

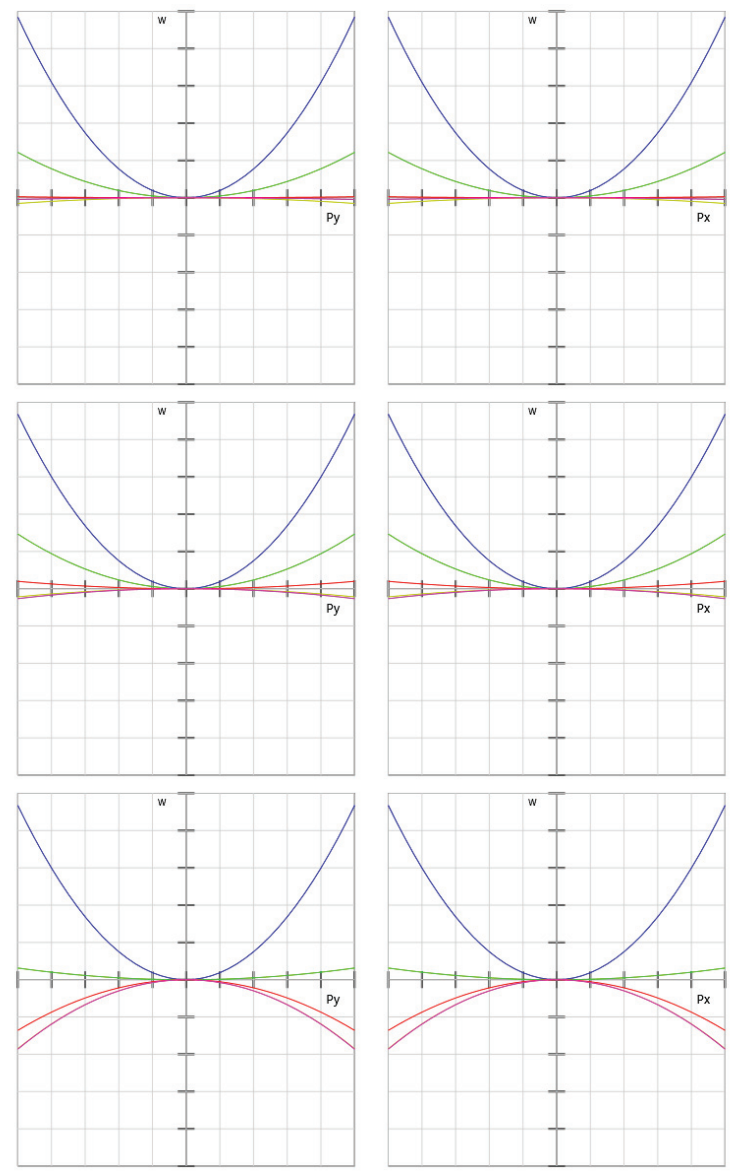

Figure 5: Optical path difference in the tangential and sagittal plane for a nominal $125 \mathrm{~mm}$ focal distance lens for varying effective refractive indices.

Evaluation at $100 \%, 50 \%$ and $25 \%$ (top to bottom) of the refractive index difference. Horizontal scale is the normalized pupil coordinate, vertical scale is in number of waves. Top graph 4 waves /div, middle graph 2 waves/div, bottom graph 0.4 wave/div. Wavelengths color coded as in figure 4 .

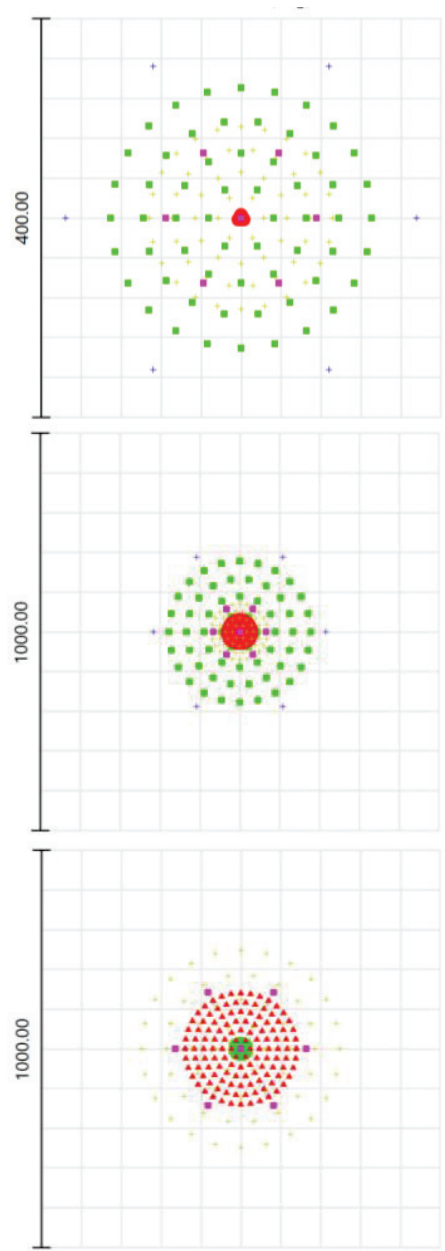

Figure 6: Spot diagram for a nominal $125 \mathrm{~mm}$ focal distance lens for varying effective refractive indices.

Evaluation at $100 \%, 50 \%$ and $25 \%$ (top to bottom) of the refractive index difference. Scale in micrometers. Wavelengths color coded as in figure 4. 